far as the Fermi interactions for oxygen and nitrogen are concerned. The reasonableness of the approximations is indicated by the agreement of the nitrogen $\mathrm{hfs}$ in both molecules (see Table I). The values of the hfs coupling constants of the observable nuclear spin splittings in the organic radical are listed in Table II.

Using the results of YSHL we have calculated the $\mathrm{O}^{17}$ isotropic hfs in nitric oxide. It is given by the same mechanism involved in the nitrogen hfs; predominantly

TABLE I. Hyperfine structure for oxygen-17.

\begin{tabular}{lcccc}
\hline Molecule & \multicolumn{2}{c}{$\begin{array}{l}\mathrm{O}^{17} \mathrm{hfs} \times 10^{24} \mathrm{~cm}^{-3} \\
\text { exp. }\end{array}$} & calc. & \multicolumn{2}{c}{$\begin{array}{c}\mathrm{N}^{14} \mathrm{hfs} \times 10^{24} \mathrm{~cm}^{-3} \\
\text { exp. }\end{array}$} & calc. \\
\hline $\mathrm{O}^{16} \mathrm{O}^{17}$ & 1.26 & $1.34,1.17$ & $\ldots$ & $\ldots$ \\
$\mathrm{N}^{14} \mathrm{O}^{17}$ & $\ldots$ & 0.63 & 0.85 & 0.71 \\
$\mathrm{R}_{2} \mathrm{~N}^{14} \mathrm{O}^{17}$ & 0.617 & $\ldots$ & 0.79 & $\ldots$ \\
\hline
\end{tabular}

from cross terms, in the configuration interaction wave function, between the ground state $(2 \pi)$ and various "excited" states having one $(2 \pi)$ and two $(\sigma)$ unpaired electrons. The $(\sigma)$ orbitals are composed of $1 s, 2 s$, and $2 p$ functions along the $\mathrm{N}-\mathrm{O}$ bond. The agreement between the calculated splitting for $\mathrm{O}^{17}$ and that observed in the model compound is quite good. It is also interesting to compare these results with those found for molecular oxygen. ${ }^{1,6,7}$ In Table I the hfs due to $\mathrm{O}^{17}$ as well as that due to $\mathrm{N}^{14}$ are listed for the three examples. The electronic structure of molecular oxygen has been studied by Kotani, Mizuno, Kayama, and Ishiguro, who find $|\psi(0)|^{2}$ given predominantly by

TABLE II. Hyperfine structure constants in di-sec-butyl nitric oxide, $g=2.00585$, in benzene solution.

\begin{tabular}{ccc}
\hline Nucleus & $A$ (gauss) & $\left|\psi\left(r_{N}=0\right)\right|^{2} \times 10^{24} \mathrm{~cm}^{-3}$ \\
\hline $\mathrm{N}^{14}$ & 13.39 & 0.79 \\
$\mathrm{H}^{1}$ & 3.84 & 0.0163 \\
$\mathrm{C}^{13}$ & 9.73 & 0.164 \\
$\mathrm{O}^{17}$ & 19.71 & 0.617 \\
\hline
\end{tabular}

cross terms containing sigma orbitals from the ground (two unpaired spins) and "excited" (four unpaired spins, etc.) configurations. ${ }^{7}$ The value of the Fermi constant is about twice that observed in di-sec-butyl nitric oxide.

The experimental arrangement used in the present work consisted of a conventional $100-\mathrm{kc}$ modulated EPR spectrometer operating at $9500 \mathrm{Mc} / \mathrm{sec}$. A homogeneous 12-in. magnet was used. The time constant at the output of the $100-\mathrm{kc}$ lock-in detector was 10 to 30 sec, when using a field sweep of $3 \mathrm{G} / \mathrm{min}$. The linewidth is about $1 \mathrm{G}$. Magnetic-field measurements were made using a proton marginal oscillator whose frequency was determined by a Hewlett-Packard $524 \mathrm{C}$ counter. The microwave frequency was determined using a HewlettPackard 540B transfer oscillator.

A more complete discussion of this particular organic radical and its relation to others in the series will be published in another paper.

We would like to thank K. Hijikata, and C. C. Lin of the University of Oklahoma, and B. Kirtman of the University of California for several helpful discussions, and D. R. Herschbach for his hospitality at the University of California during part of this work.

\footnotetext{
* Permanent address: Department of Chemistry, Brown University, Providence, Rhode Island,

is. L. Miller and C. H. Townes, Phys. Rev. 90, 537 (1953).

$2 \mathrm{H}$. Brion, C. Moser, and M. Yamazaki, J. Chem. Phys. 30, 673 (1959)

${ }^{3}$ C. C. Lin, K. Hijikata, and M. Sakamoto, J. Chem. Phys. 33, 878 (1960).

${ }^{4}$ M. Yamazaki, M. Sakamoto, M. Hijikata, and C. C. Lin, J. Chem. Phys. 34, 1926 (1961).

5 J. C. Baird and J. R. Thomas, J. Chem. Phys. 35, 1507 (1961).

${ }^{6}$ S. L. Miller, C. H. Townes, and M. Kotani, Phys. Rev. 90, $542(1953)$

${ }^{7}$ M. Kotani, Y. Mizuno, K. Kayama, and E. Ishiguro, J. Phys. Soc. Japan 12, 707 (1957).
}

\section{Extrema in Velocity Dependence of Total Elastic Cross Sections for Atomic Beam Scattering: Relation to Di-atom Bound States $\dagger$}

\author{
RICHARD B. BERNSTEIN \\ Chemistry Department, University of Michigan, \\ Ann Arbor, Michigan
}

(Received July 31, 1962)

$\mathbf{U}$ NDULATORY deviations from the Massey-Mohr (MM) dependence of the total elastic cross section $Q(v)$ for atom-atom scattering have been predicted ${ }^{1}$ and observed. ${ }^{2}$ The present communication indicates (1) a relationship between the maxima in $Q(v)$ and the number of stable di-atom bound states, and (2) a procedure for evaluating the potential from the extrema velocities. A single, ${ }^{3}$ static, central potential is assumed. The principles of the present treatment are general and are applicable to any "realistic" potential [long-range inverse power $(s \geq 3)$ attraction, steep short-range repulsion]; for the purpose of concise illustration, however, the LJ (12-6) potential is employed here, using the notation of reference 1.

The extrema in $Q(v)$ are a consequence of the broad maximum in $\eta(l)$, which provides a significant number of nonrandom phases. ${ }^{1}$ The maximum phase $\eta^{m}$ increases with decreasing $v$ and, assuming the attractive potential is sufficiently strong, can pass successively through $\pi / 2, \pi$, etc., yielding substantial positive and negative deviations from $Q_{M M}$ (calculated via the random-phase approximation). 
We index the extrema in $Q$ from high to low velocity, starting with $N=1$ for the first maximum, $N=1.5$ for the minimum which follows, etc., to $N=M$ for the maximum at lowest $v$. Analysis of the MM derivation reveals that the condition for greatest deviation from $Q_{M M}$ is $\eta_{N}^{m}=\pi\left(N-\frac{1}{4}\right)$. A semiclassical treatment ${ }^{4}$ shows that the maximum reduced phase depends solely upon $K \equiv \frac{1}{2} \mu v^{2} / \epsilon$, increasing monotonically with decreasing $K$. Also, $l_{m}$ decreases toward zero as $k \equiv \mu v / \hbar$ decreases, so that $\eta^{m}(k)$ approaches $\eta_{0}(0)$. In the lowenergy region the semiclassical approximation loses validity, and a partial wave analysis ${ }^{1}$ is required. We note that, rigorously, $\eta^{m}(0)<\eta_{0}(0)+\pi / 2$; in addition, considerations based on scattering-length theory

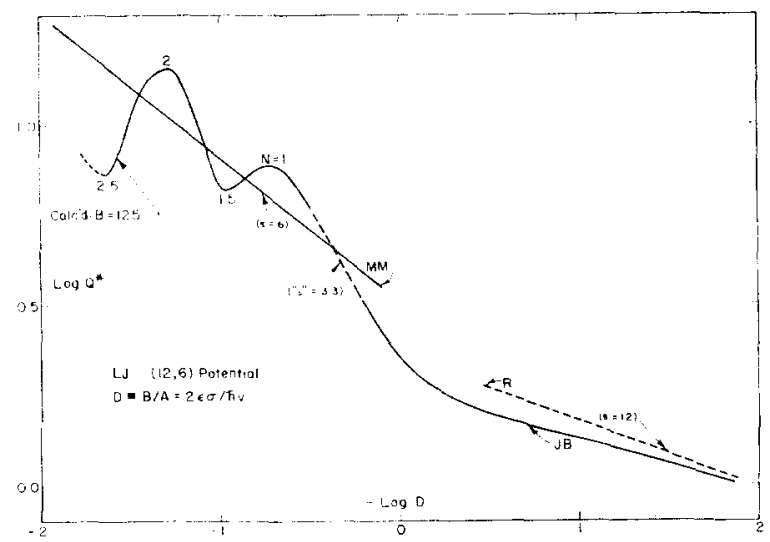

Fig. 1. Velocity dependence of the cross section.

strongly suggest ${ }^{5}$ that $\eta^{m}(k)<\eta^{m}(0)$. By Levinson's theorem ${ }^{6} \eta_{0}(0)=n \pi$, where $n$ is the number of discrete levels of zero angular momentum. Thus $N<n+\frac{3}{4}$, so that $M=n$; i.e., the number of maxima in $Q(v)$ equals the number of bound vibrational states of the di-atom.

Returning to the semiclassical analysis, ${ }^{4}$ we obtain for the LJ $(12,6)$ potential $\eta^{m}=(120 \pi / 847)(231 / 160)^{1 / 6}$ $D \cdot g(K)$, where $D \equiv B / A=2 \epsilon \sigma / \hbar v$ and $g(K)=1-$ $0.25 K^{-1}+\cdots \cong 1-0.25 D B^{-\frac{1}{2}}$ (valid for $D \leq 2 B^{\frac{1}{2}}$ ). With the previous formula for $\eta_{N}{ }^{m}$ this yields $N-\frac{1}{4}=0.3012$ $(\epsilon \sigma / \hbar) v_{N}{ }^{-1}\left[1-0.354(\epsilon / \mu)^{\frac{2}{2}} v_{N}{ }^{-1}\right]$, where $v_{N}$ is the velocity of the $N$ th extremum. A plot of $N-\frac{1}{4}$ vs $v_{N}{ }^{-1}$ must pass through the origin; this serves to verify the assignment of the indices to the extrema. The initial slope of the line yields $\epsilon \sigma$; the curvature is governed by $\epsilon / \mu$. In addition, one may plot $\left(N-\frac{1}{4}\right) v_{N}$ vs $v_{N}{ }^{-1}$; the ratio of slope to intercept yields separately $\epsilon$, but with low sensitivity.

Consider next the "high-velocity" region (criterion: $\left.\eta^{m}<\frac{1}{2}\right)$. Using the Jeffreys-Born (JB) approximation for the LJ $(12,6)$ phase $^{1}$ one obtains, upon rederiving the MM equation

$$
\begin{aligned}
Q_{\mathrm{JB}}{ }^{*}= & \beta_{1}{ }^{2}\left\{2+(1 / 10)(63 \pi / 128)^{2} D^{2} \beta_{1}{ }^{-22}\right. \\
& \left.\times\left[1-(640 / 147) \beta_{1}{ }^{6}+(2560 / 441) \beta_{1}{ }^{12}\right]\right\},
\end{aligned}
$$

valid for $D \leq 1$, and usable as an approximation (within a few percent) to $D \cong 3$. Here $\beta_{1}$ is the root of $\beta^{11}+$

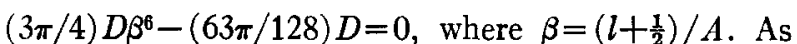
$D \rightarrow 0$, asymptotically $\beta_{1} \sim 1.0404 D^{1 / 11}$ and $Q_{\mathrm{R}}{ }^{*} \sim$ $(21 / 10)(63 \pi / 128)^{2 / 11} D^{2 / 11}=2.273 D^{2 / 11}$, as expected for an inverse power $(s=12)$ repulsive potential. This is to be compared with $Q_{M M}{ }^{*}=3.170 D^{2 / 5}$ ( $s=6$ attraction only $\left.^{1}\right)$. Figure 1 shows a graph of Eq. (1), labeled JB; the straight lines $\mathrm{R}$ and $\mathrm{MM}$ designate $Q_{\mathrm{R}}{ }^{*}$ and $Q_{\mathrm{M}}{ }^{*}$. Shown also is an example of a curve of $Q^{*}$ in the "lowvelocity" region $(D \geq 3)$, calculated ${ }^{1}$ via the standard partial wave procedure, for $B=125$. The "wavelength" of the undulatory deviations decreases with increasing $\epsilon \sigma$, making resolution more difficult. Further discussion, including a consideration of the "amplitude" of the undulations (which decreases with increasing $\mu$ ), is deferred to a detailed paper in preparation.

Applying the present procedures to the data of reference 2 , one obtains for $\mathrm{Li}-\mathrm{Xe}$ and $\mathrm{K}-\mathrm{Xe}$ values for $10^{22} \epsilon \sigma(\mathrm{erg} \mathrm{cm})$ of $9.4_{5}$ and $6.7_{2}$, respectively. Indexing of the extrema thus far observed ${ }^{2}$ implies a minimum of three vibrational states each for the $\mathrm{LiXe}$ and $\mathrm{KXe}$ molecules in the ${ }^{2} \Sigma^{+}$state.

† Supported by the U. S. Atomic Energy Commission Div. of Research.

${ }^{1}$ R. B. Bernstein, J. Chem. Phys. 34, 361 (1961); 33, 795 (1960).

${ }^{2}$ E. W. Rothe, P. K. Rol, S. M. Trujillo, and R. H. Neynaber, Phys. Rev. (to be published).

${ }_{3}$ For simplicity we limit consideration to unlike atom collisions involving one ${ }^{1} S_{0}$ state atom (Group II or VIII) and one either ${ }^{1} S_{0}$ or ${ }^{2} S_{i}$ (Group I), yielding the single molecular state ${ }^{1} \Sigma^{+}$or ${ }^{2+} \Sigma^{+}$

${ }^{4}$ R. B. Bernstein, J. Chem. Phys. 36, 1403 (1962)

${ }^{5}$ It has not been possible to prove this in general, however. Thus in unfavorable circumstances it is possible that $M=n+1$.

${ }^{6}$ See P. Swan, Proc. Roy. Soc. (London) A228, 10 (1955); in the present case $^{3}$ Pauli excluded states are not involved.

\section{Alternating Linewidths in the ESR Spectra of Dinitrobenzene Anion Radicals*}

Jack H. Freed, $†$ Philip H. Rieger, $\$$ and George K. Fraenkel Department of Chemistry, Columbia University New York 27, New York

(Received July 5, 1962)

$\mathbf{W}$ $\mathrm{E}$ have found an alternation in the linewidths in the ESR spectrum of the anion radical of $m$ dinitrobenzene. The radical was produced by electrolytic reduction in $\mathrm{N}, \mathrm{N}$-dimethyl-formamide solution at room temperature. The derivative spectrum obtained at $-50^{\circ} \mathrm{C}$ shows that those hyperfine components corresponding to $m_{\mathrm{N}}= \pm 1$ (where $m_{\mathrm{N}}$ is the total z component of the nitrogen nuclear spin angular momentum) are considerably broader and of smaller amplitude than those for which $m_{\mathrm{N}}= \pm 2.0$. At room temperature, the effect is much weaker but may be detected by careful analysis of the spectrum. Examination of the spectra of the $p$ - and $o$-dinitrobenzene 